\title{
The no-longer uncharted territory
}

Results of a genome-wide, collaborative effort to characterize the mouse transcriptome have been published. They reveal important information about the extent and the complexities of transcription.

The two recently published reports describe data from FANTOM3 (FANTOM stands for Functional Annotation of the Mouse), which is an international collaboration led by RIKEN, Japan. The group started by combining several approaches, including CAGE (new cap analysis gene expression) and two ditag technologies named as GIS/GSC (GIS, gene identification signature; GSC, gene signature cloning), to identify transcriptional start sites and termination sites. Corresponding pairs of these sites were identified for 181,047 independent transcripts - this number is an order of magnitude greater than the estimated number of genes in the mouse genome. The discrepancy can be attributed, at least in part, to the fact that alternative promoters and polyadenylation sites are associated with most transcriptional units. And at least $65 \%$ of transcriptional units contain several splice variants.

More than a third of the cDNAs in the FANTOM3 data set represent non-coding RNAs. The authors find that although non-coding RNAs are less conserved than $5^{\prime}$ and $3^{\prime}$ UTRs, their promoters are more conserved than those of protein-coding RNAs.

Mouse-human comparison revealed that human transcriptome is comparably complex. The authors point out that the abundance of transcriptional units and the overlap between them has implications for interpreting results from microarray and genome manipulation studies.

The FANTOM3 data also indicate that antisense transcription is more widespread than previously thought: $72 \%$ of transcriptional units overlap, at least partially, with a unit on the opposite strand. Co-expressed sense-antisense pairs show complex and tissue-specific regulation. Intriguingly, the authors find that expression of these pairs frequently co-varies - this is the opposite of what would be expected if the antisense transcript negatively regulated sense transcription. The authors suggest that "[i]f antisense transcripts do reflect the transcriptional activity of enhancers, the act of transcription from the antisense promoter may generate the regulatory interaction."

Although mouse transcriptome characterization will continue, it is already clear that the transcriptome landscape holds many surprises in store. We are only beginning to get our bearings.

\footnotetext{
Magdalena Skipper

(4) References and links ORIGINAL RESEARCH PAPERS The FANTOM Consortium and RIKEN Genome Exploration Research Group and Genome Science Group. The transcriptional landscape of the mammalian genome. Science 309, 1559-1563 (2005) | The FANTOM Consortium and RIKEN Genome Exploration Research Group and Genome Science Group. Antisense transcription in the mammalian genome. Science 309, 1564-1566 (2005)
}

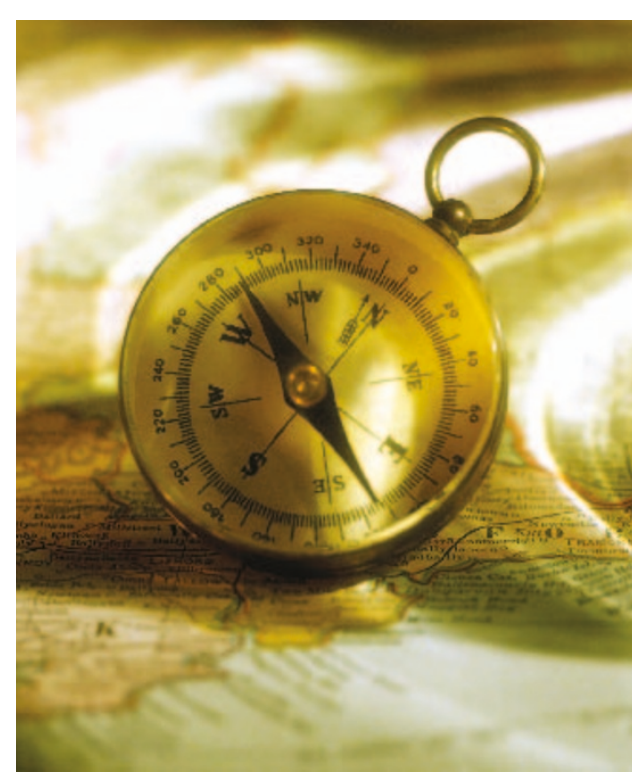

